\title{
Utility Max-Min Flow Control Using Slope-Restricted Utility Functions
}

\author{
Jeong-woo Cho, Member, IEEE, and Song Chong, Member, IEEE
}

\begin{abstract}
We present a network architecture for the distributed utility max-min flow control of elastic and nonelastic flows where utility values of users (rather than data rates of users) are enforced to achieve max-min fairness. The proposed link algorithm converges to utility max-min fair bandwidth allocation in the presence of round-trip delays without using the information of users' utility functions. To show that the proposed algorithm can be stabilized not locally but globally, we found that the use of nonlinear control theory is inevitable. Even though we use a distributed flow-control algorithm, it is shown that any kind of utility function can be used as long as the minimum slopes of the functions are greater than a certain positive value. Though our analysis is limited to the single-bottleneck and homogeneous-delay case, we believe that the proposed algorithm is the first to achieve utility max-min fairness with guaranteed stability in a distributed manner.
\end{abstract}

Index Terms-Absolute stability, delay systems, flow control, nonlinear systems.

\section{INTRODUCTION}

$\mathbf{O}$ NE OF THE MOST common understandings of fairness for a best-effort service network is max-min fairness as defined in [1]. An easy algorithm for obtaining a max-min fair allocation, which is also known as a waterfilling algorithm, is given in [1] and [2]: rates of flows are increased at the same pace until a link is saturated. Then the rates of flows passing through the saturated link are fixed, and others continue to increase at the same pace. There are several works [3]-[6] that provide distributed and stable max-min flow-control algorithms that work in multiple-bottleneck networks in spite of round-trip delays. (In this paper, we do not deal with other concepts of fairness [7]-[9]).

The rapid growth of multimedia applications has triggered a new fairness concept: utility max-min fairness. The definition of utility max-min fairness is similar to that of bandwidth max-min fairness, except that utility values of users are max-min fair. In a single-link case, utility max-min corresponds to the satisfaction (utilities) of each user in the network being equal. Let us consider a simple network in which a link of capacity $\mu$ is shared by two flows: an elastic flow with utility function $U_{1}(\cdot)$ and a real-

Paper approved by T.-S. P. Yum, the Editor for Packet Access and Switching of the IEEE Communications Society. Manuscript received July 18, 2005; revised June 20, 2006. This work was supported by the Center for Broadband OFDM Mobile Access (BrOMA) at POSTECH through the ITRC program of the Korean MIC, supervised by IITA under IITA-2006-C1090-0603-0037. This paper was presented in part at the IEEE Global Telecommunications Conference (GLOBECOM), St. Louis, MO, November 2005.

J. Cho is with the Telecommunication R\&D Center, Samsung Electronics, Suwon 442-600, Korea (e-mail: ggumdol@gmail.com).

S. Chong is with the School of Electrical Engineering and Computer Science, Korea Advanced Institute of Science and Technology, Daejeon 305-701, Korea (e-mail: song@ee.kaist.ac.kr).

Digital Object Identifier 10.1109/TCOMM.2007.896066

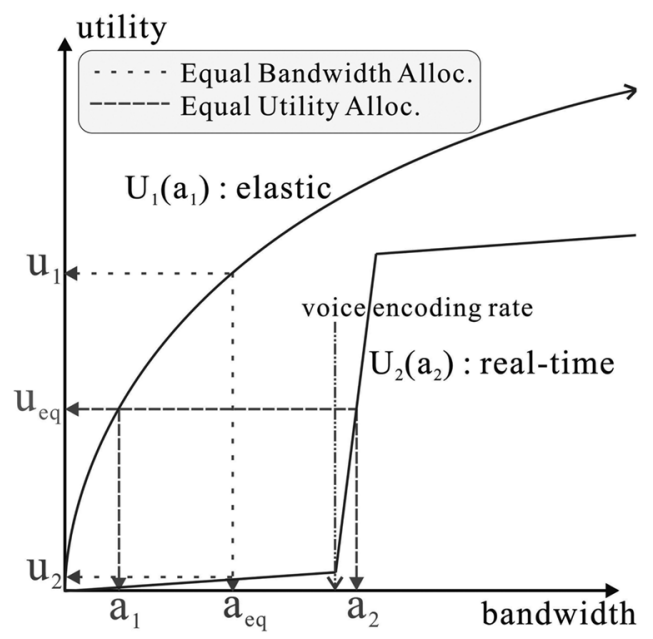

Fig. 1. Bandwidth max-min fairness versus utility max-min fairness.

time flow that transfers voice data with utility function $U_{2}(\cdot)$. As shown in Fig. 1 , if the link capacity is shared equally (i.e., $a_{\mathrm{eq}}=$ $(\mu / 2))$, the utility of the elastic flow, $u_{1}\left(=U_{1}\left(a_{\mathrm{eq}}\right)\right)$, becomes much larger than that of the real-time flow, $u_{2}\left(=U_{2}\left(a_{\mathrm{eq}}\right)\right)$, and the real-time flow is unsatisfactory because the allowed rate is smaller than the voice encoding rate. In contrast, if the link capacity is shared in a way that $U_{1}^{-1}\left(u_{\text {eq }}\right)+U_{2}^{-1}\left(u_{\text {eq }}\right)=\mu$, then both flows gain an identical utility (i.e., $U_{1}\left(a_{1}\right)=U_{2}\left(a_{2}\right)=$ $u_{\text {eq }}$ ), and the real-time flow is satisfied with the allocation because the allowed rate is greater than the voice encoding rate. The former represents the bandwidth max-min fair allocation (equal bandwidth allocation in the single-link case), whereas the latter represents the utility max-min fair allocation (equal utility allocation in the single-link case).

\section{A. Related Works}

Originally, Bianchi et al. [10] motivated application performance-oriented flow control in wireless networks by proposing utility-fair bandwidth allocation where application-specific quality-of-service (QoS) of flows are satisfied. They also presented a centralized algorithm that can equalize utilities of flows, assuming utility functions are piecewise-linear. Cao and Zegura [11] introduced a broader conception, utility max-min fairness. They emphasized that applications have various kinds of utility function in general. For example, a voice over IP (VoIP) user corresponds to a step-like utility function, because his satisfaction is at a maximum if the allowed rate is larger than the voice encoding rate, and is at a minimum if the allowed rate is smaller than the encoding rate. The satisfaction of teleconference users with multilayer streams consisting of a base-layer stream and multiple enhancement-layer streams would incrementally increase as 
additional layers were allowed. Therefore, to accommodate various types of application, it is necessary to relax the restriction on the shapes of utility functions as much as possible.

Regarding the characteristics of the utility max-min fairness, two works are remarkable: Radunović and LeBoudec [2] pointed out that there are some cases to which the notion of a bottleneck link and the waterfilling approach is not applicable. They considered not only max-min, but also min-max fairness, and observed that the existence of max-min fairness is actually a geometric property of the set of feasible allocations. Based on the relation between max-min fairness and leximin ordering, a concept they borrowed from economy, they completed a unified framework encompassing weighted and unweighted max-min fairness and utility max-min fairness, and provided a centralized algorithm that yields these fairness properties. Sakar and Tassiulas [12] found that a utility max-min fair allocation does not necessarily exist when we relax the assumptions that utility functions are continuous and strictly increasing. To complete a consistent fairness concept, they introduced a maximally fair utility allocation that complements the utility max-min fair allocation. They also presented an algorithm for computing the allocation in polynomial number of iterations. In the meantime, Harks and Poschwatta [13], [14] introduced the concept of utility proportional fairness, where utilities' values of flows are enforced to achieve proportional fairness. They presented distributed algorithms to achieve the fairness although they did not consider the stability in the presence of round-trip delays.

There are several works [2], [11], [15] that present link algorithms to achieve the utility max-min fair bandwidth allocation, assuming that each link knows the utility functions of all the flows sharing the link. Note that the algorithms used in the cited studies are not distributed in the strict sense, because they require global information, such as utility functions of users. Wydrowski et al. [16] proposed a somewhat similar architecture, although they did not mention utility max-min fairness. They considered a linearized model in which even gain values depend on the equilibrium point, which cannot be known in advance. Note that utility functions are naturally nonlinear, and local stability results obtained through linearization techniques cannot guarantee global stability. It is very difficult to find a region of attraction [17] in such works.

\section{B. Our Contribution}

After we examine related works, two questions remain: 1) whether or not there exists a distributed link algorithm that does not require per-flow information, including utility function information; and 2) whether or not such an algorithm converges in the presence of round-trip delays. As a solution to these questions, we provide a network architecture with a distributed flowcontrol algorithm that achieves utility max-min fairness without using any kind of per-flow operations, and provides stability results for the proposed flow-control algorithm. In our proposed architecture, links do not need to know the utility functions of flows sharing the links.

It should also be noted that we consider a nonlinear model that does not exploit knowledge of the equilibrium point. To the best of our knowledge, this is the first work dealing with an analytical framework for the original problem and its global stability.

\section{Paper Organization}

The rest of this paper is organized as follows. In Section II, we provide a formal definition of utility max-min fairness and our proposed network architecture for utility max-min flow control. In Section III, we provide stability results based on Dewey and Jury's [20] criterion for our proposed systems. We provide a method for estimating the number of flows bottlenecked at a link, and give emphasis to the applicability of our work in Section IV. After presenting several simulation results with a multiple-bottleneck network in Section V, we conclude.

\section{UtILITY MAX-Min ARCHITECTURE}

To introduce a formal definition of the utility max-min fairness, let us denote flow $i$ 's utility value and utility function by $u_{i}$ and $U_{i}(\cdot)$, respectively. Two technical assumptions on $U_{i}(\cdot)$ for the analysis of the proposed network architecture are given as follows.

[A.1] We assume that $U_{i}(\cdot)$ is a continuous and increasing function of user $i$ 's allocated data rate. By this assumption, there always exists an inverse function of $U_{i}(\cdot)$, i.e., $U_{i}^{-1}(\cdot)$. It is quite natural that the values of utility functions increase as the allocated data rates increase. Note that this assumption does not hold for all utility functions [12].

[A.2] We assume that $U_{i}(0)=0$. It is also quite reasonable, since the utility function value of user $i$, i.e., the degree of user $i$ 's satisfaction, is zero when zero data rate is allocated.

Let us denote the set of all links, the set of all flows, and the set of flows traversing link $l$ by $L, N$, and $N(l)$, respectively. Their cardinalities are denoted by $|L|,|N|$, and $|N(l)|$, respectively. Then, similar to the bandwidth max-min fairness [1], the utility max-min fairness can be defined as follows.

Definition 1: A rate vector $\left\langle a_{1}, \ldots, a|N|\right\rangle$ is said to be feasible if it satisfies $a_{i} \geq 0, \forall I \in N$ and $\sum_{i \in N(l)} a_{i} \leq \alpha_{t}^{l} \mu^{l}$ $\forall L \in L$.

Definition 2: A rate vector $\left\langle a_{1}, \ldots, a_{|N|}\right\rangle$ is said to be utility max-min fair if it is feasible, and for each $i \in N$ and feasible rate vector $\left\langle\bar{a}_{1}, \ldots, \bar{a}_{|N|}\right\rangle$ for which $U_{i}\left(a_{i}\right)<U_{i}\left(\bar{a}_{i}\right)$, there exists some $i^{\prime}$ with $U_{i}\left(a_{i}\right) \geq U_{i^{\prime}}\left(a_{i^{\prime}}\right)>U_{i^{\prime}}\left(\bar{a}_{i^{\prime}}\right)$.

Here $\mu^{l}$ denotes the capacity of link $l$, and $\alpha_{T}^{l}$ is a constant defining target link use of link $l\left(0<\alpha_{T}^{l} \leq 1\right)$. Let a vector $\left\langle u_{1}, \ldots, u_{|N|}\right\rangle$ denote the utility vector corresponding to the rate vector $\left\langle a_{1}, \ldots, a_{|N|}\right\rangle$ where $u_{i}=U_{i}\left(a_{i}\right) \forall i \in N$. Then, Definition 2 can be restated more informally as follows: a rate vector $\left\langle a_{1}, \ldots, a_{|N|}\right\rangle$ is said to be utility max-min fair if it is feasible and for each user $i \in N$, its utility $u_{i}$ cannot be increased while maintaining feasibility without decreasing the utility $u_{i^{\prime}}$ for some user $i^{\prime}$, for which $u_{i^{\prime}} \leq u_{i}$.

We propose a network architecture that achieves utility max-min fairness at equilibrium. The network architecture with multiple sources and links is depicted in Fig. 2. Let us consider a bottleneck link $l \in L$. Then, the dynamics of the buffer of the link can be written as

$$
\dot{q}^{l}(t)= \begin{cases}\sum_{i \in N(l)} a_{i}\left(t-\tau_{i}^{l, f}\right)-\mu^{l}, & q^{l}(t)>0 \\ {\left[\sum_{i \in N(l)} a_{i}\left(t-\tau_{i}^{l, f}\right)-\mu^{l}\right]^{+},} & q^{l}(t)=0\end{cases}
$$




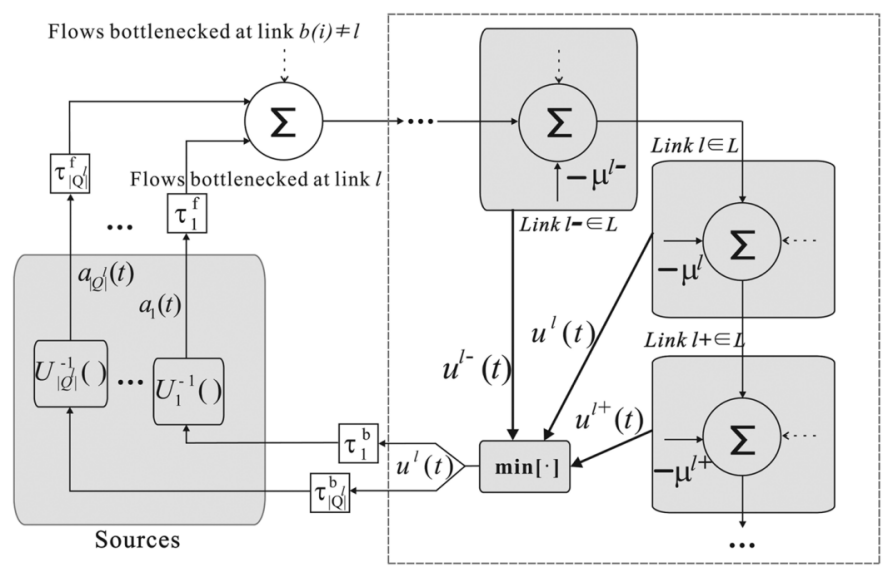

Fig. 2. Network architecture for utility max-min fairness.

where $a_{i}(t)$ is the sending rate of source $i, \tau_{i}^{l, f}$ is the forwardpath delay from source $i$ to link $l, \mu^{l}$ is the link capacity of the link, and the saturation function $[\cdot]^{+} \triangleq \max [\cdot, 0]$ is such that $q^{l}(t)$ cannot be negative.

Source $i$ sends packets at a data rate of $a_{i}(t)$. There is one reserved field in every packet that carries the current utility value. The initial value is replaced with a sufficiently large value. When a packet reaches a link along the forward path of its flow, each link replaces the field value with its utility value if its utility value is smaller than the field value. In this way, the smallest one among utility values of links along the forward path of flow $i$ is delivered to source $i$. Thus we assume the following source algorithm:

$$
\text { Source Algorithm : } a_{i}(t)=U_{i}^{-1} \underbrace{\left(\min _{l \in L(i)}\left[u^{l}\left(t-\tau_{i}^{l, b}\right)\right]\right)}_{u_{i}(t) \triangleq}
$$

where $L(i)$ is the set of links which flow $i$ traverses, $u^{l}(t)$ is the utility value assigned by link $l$ on the path of flow $i, \tau_{i}^{l, b}$ is the backward-path delay from link $l$ to source $i$, and $U_{i}(\cdot)$ is the user-specific utility function of user $i$. Because the min[-] operation is taken over a finite number of links, there should exist at least one link $l$ such that $u^{l}=\min [\cdot]$. Therefore, each flow $i$ has at least one bottleneck $l \in L(i)$.

There are two assumptions employed for the analysis of the network model.

[B.1] We assume that the sources are persistent until the closed-loop system reaches a steady state. By "persistent," we mean that the source always has enough data to transmit at the allocated rate.

[B.2] Two delays, say, the forward-path delay $\tau_{i}^{l, f}$ and the backward-path delay $\tau_{i}^{l, b}$, include propagation, queueing, and transmission and processing delays. We denote the sum of two delays by $\tau_{i}$ and assume that this is constant. In general, stability analysis of nonlinear systems with timevarying delays requires the application of Razumikhin theorems [18] which are known to be abstruse.

\section{A. PID and Proportional-Integral-Double-Integral ${ }^{2}\left(P I I^{2}\right)$ Link Controller Models}

To control flows and to achieve utility max-min fairness, we use a proportional-integral-derivative (PID) link controller at each link. In the PID link controller model, there is a specified target queue length $q_{T}^{l}$ to avoid underuse of the link capacity. Because we have a nonzero target queue length $q_{T}^{l}$, the PID model implies that $\alpha_{T}^{l}=1$ in Definition 1. Each link calculates the common feedback utility value $u^{l}(t)$ for all flows traversing the link according to the PID control mechanism.

In general, a proportional term increases the convergence speed of transient responses and reduces errors caused by disturbances. An integral term effectively eliminates steady-state error and results in the size of the stability region being reduced. A derivative term adds some damping and extends the area of the stability region. It also improves the performance of the transient period.

Let us denote the set of flows bottlenecked at link $l$ and its cardinality by $Q^{l}$ and $\left|Q^{l}\right|$, respectively. The link algorithm with PID controller that uses the difference between $q^{l}(t)$ and $q_{T}^{l}$ as input is given by the Link Algorithm 1 described by (LA1), where $e_{1}^{l}(t) \triangleq q^{l}(t)-q_{T}^{l}$ is the error signal between control target and current output signal, and $g_{P}>0$ and $g_{I}, g_{D} \geq 0$. It should be noted that we also can use a $\mathrm{PII}^{2}$ controller as we did in [19], by defining $e_{2}^{l}(t) \triangleq \sum_{i \in N(l)} a_{i}\left(t-\tau_{i}^{l, f}\right)-\alpha_{T}^{l} \mu^{l}$ where $\alpha_{T}^{l}<1$. The link algorithm with the PII $^{2}$ controller is given by the Link Algorithm 1 in (LA2). See (LA1) and (LA2) at the bottom of the page.

This link algorithm controls flows so that the queue length at steady state becomes zero at the cost of link underuse. The main advantage of this model is that the feedback signal is not saturated at $q^{l}(t)=0$, and it is shown through simulations in [19] that the $\mathrm{PII}^{2}$ model results in faster convergence. In this paper, though we focus on the PID model to avoid repeating similar arguments for the $\mathrm{PII}^{2}$ model, readers should note that one can derive similar arguments regarding the $\mathrm{PII}^{2}$ model with ease, as was done in [19].

Link Algorithm 1: $u^{l}(t)=\left[-\frac{1}{\left|Q^{l}\right|}\left(g_{P} e_{1}^{l}(t)+g_{I} \int_{0}^{t} e_{1}^{l}(t) d t+g_{D} \dot{e}_{1}^{l}(t)\right)\right]^{+}$

Link Algorithm 2: $u^{l}(t)=\left[-\frac{1}{\left|Q^{l}\right|}\left(h_{P} e_{2}^{l}(t)+h_{I} \int_{0}^{t} e_{2}^{l}(t) d t+h_{I^{2}} \int_{0}^{t} \int_{0}^{t} e_{2}^{l}(t) d t d t\right)\right]^{+}$ 

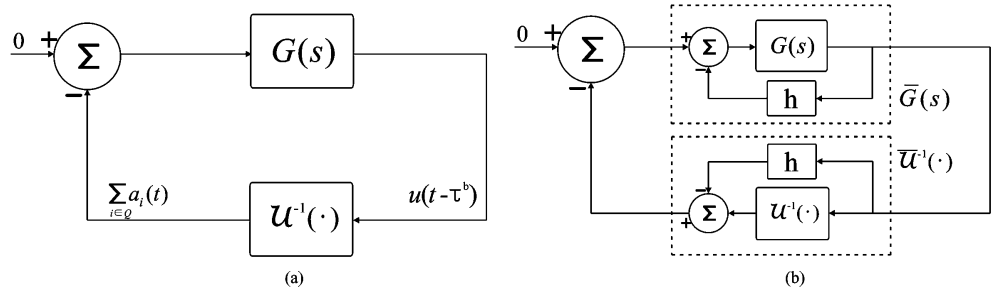

Fig. 3. Block diagrams of the proposed architecture. (a) Original. (b) After loop transformation.

\section{B. Steady-State Analysis}

Suppose that the closed-loop system has an equilibrium point, i.e., $q^{l}(t) \rightarrow q_{T}^{l}, u^{l}(t) \rightarrow u^{l *}$, and $a_{i}(t) \rightarrow a_{i}^{*}$. More formally, the set of flows bottlenecked at link $l$ is given by

$$
Q^{l}=\left\{i \in N(l) \mid a_{i}^{*}=U_{i}^{-1}\left(u^{l *}\right)\right\}
$$

and the set of all flows not bottlenecked at link $l$ but traversing link $l, N(l)-Q^{l}$, is given by

$$
N(l)-Q^{l}=\left\{i \in N(l) \mid a_{i}^{*}<U_{i}^{-1}\left(u^{l *}\right)\right\} .
$$

Then (1) implies that the link capacity $\mu^{l}$ in the PID link controller model is fully used as follows:

$$
\sum_{i \in N(l)} a_{i}^{*}=\mu^{l}
$$

Using (4), and the definitions (2) and (3), we obtain

$$
\sum_{i \in Q^{l}} U_{i}^{-1}\left(u^{l *}\right)+\sum_{i \in N(l)-Q^{l}} U_{i}^{-1}\left(u^{l^{\prime} *}\right)=\mu^{l} .
$$

Thus, we finally get the following equation, which means that flows with utility values $u^{l^{\prime} *}<u^{l *}$, which are not bottlenecked at link $l$, are assigned data rates $U_{i}^{-1}\left(u^{l^{\prime} *}\right)$ in advance, and the remaining capacity is fairly distributed to flows bottlenecked at link $l$ based on the common utility value $u^{l *}$

$U_{i}\left(a_{i}^{*}\right)=u^{l *}=\left[\sum_{i \in Q^{l}} U_{i}^{-1}\right]^{-1}\left(\mu^{l}-\sum_{i \in N(l)-Q^{l}} U_{i}^{-1}\left(u^{l^{\prime} *}\right)\right)$

for all $i \in Q^{l}$. From the above arguments, we can show that the proposed network architecture possesses the utility max-min fairness property. (All proofs in this paper are in the appendices.)

Theorem 1 (Utility Max-Min Fairness): The proposed network architecture described by (1), (SA), and (LA1) [or (LA2)] achieves utility max-min fairness at steady state.

\section{Stability ANALYSIS: Single-BotTLENECK AND HOMOGENEOUS-DELAY CASES}

Although we presented a multiple-bottleneck network model in Section II, rigorous stability analysis of these kinds of models was shown to be very difficult in [4], due to the dynamics coupling among links that operate on a "first come first served"
(FCFS) principle. In [4] and [19], though such dynamics coupling exists in theory, the effect of coupling was shown to be negligible through various simulations. Recently, Wydrowski $e t$ al. [16] also showed that the dynamics coupling is of a very weak form. Thus, in this section, we drop the superscript $l$, and the analysis is focused on a single-bottleneck model. We conjecture that our analytical results can be extended to multiple-bottleneck models without significant modification.

We provide a stability theorem in a single-bottleneck network, where the saturation functions employed in (1) and (LA1) are relaxed. Although our main stability theorem assumes that flows experience the same forward-path and backward-path delays, we conjecture that our theorem will hold even if flows experience heterogeneous delays, when an upper bound of $\tau_{i} s$, i.e., $\bar{\tau} \geq \max _{i \in N}\left[\tau_{i}\right]$ is used. Note that we are confident that this conjecture is true, since we have provided a similar network architecture with a distributed flow-control algorithm that achieves bandwidth max-min fairness in [19], and have proved that an analogous conjecture is really true.

To analyze the single-bottleneck and homogeneous-delay cases of the PID control model, let $\tau_{i}^{f}=\tau^{f}, \tau_{i}^{b}=\tau^{b} \forall i \in Q$ and $\tau^{f}+\tau^{b}=\tau$. Then all flows experience the same forward-path and backward-path delay. By (LA1) and (1), we obtain the following equation:

$$
\begin{array}{r}
\dddot{u}\left(t-\tau^{b}\right)=-\frac{1}{|Q|}\left(\sum_{i \in Q} g_{P} \ddot{a}_{i}(t-\tau)+g_{I} \dot{a}_{i}(t-\tau)\right. \\
\left.+g_{D} \dddot{a}_{i}(t-\tau)\right) .
\end{array}
$$

Thus we can see that the following transfer function $G(s)$, which is generated by taking the Laplace transform of the above equation, defines the relationship between $-\sum_{i \in Q} a_{i}(t)$ and $u\left(t-\tau^{b}\right)$ :

$$
G(s) \triangleq \frac{g_{P} s+g_{I}+g_{D} s^{2}}{s^{2}} \exp (-\tau s)
$$

By defining $\mathcal{U}(\cdot)$ as follows, we acquire the block diagram shown in Fig. 3(a), which is a feedback connection of $G(s)$ and an increasing and continuous nonlinearity $\mathcal{U}(\cdot)$

$$
\mathcal{U}^{-1}(u) \triangleq \frac{1}{|Q|} \sum_{i \in Q} U_{i}^{-1}(u)
$$




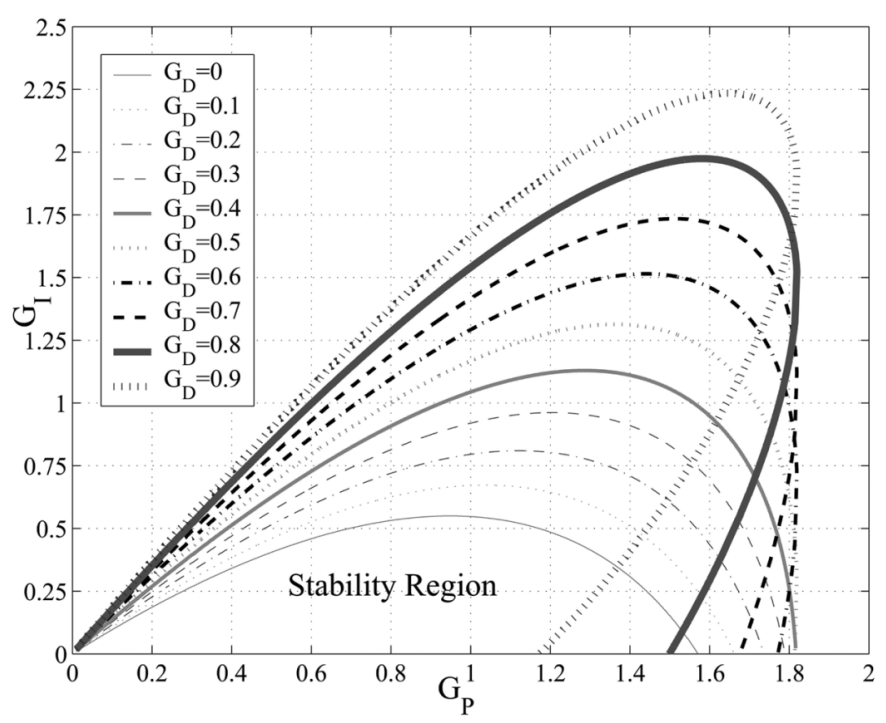

Fig. 4. Explicit stability region in terms of $G_{D}, G_{P}$, and $G_{I}$ when the feedback is an identity function.

Thus, we can expect from Fig. 3(a) that an absolute stability theorem might be applicable to the proposed closed-loop system. Of various absolute stability criteria, we have found that Dewey and Jury's criterion [20] is suitable for our systems.

The first procedure when applying this criterion is to determine whether $G(s)$ is asymptotically stable, because $G(s)$ itself without feedback is required to be asymptotically stable to apply the criterion. However, we can see that the transfer function $G(s)$ itself without feedback is not asymptotically stable, because it has a double pole at $s=0$. To overcome this problem, we use a loop transformation with a constant $h>0$, and the resulting system is shown in Fig. 3(b). It should be noted that the modified system is identical to the original system.

In our previous paper [19], it was shown the closed-loop system with feedback $\mathcal{U}^{-1}(u)=u$ (an identity function) is asymptotically stabilized if and only if (iff) the gains $G_{D} \triangleq g_{D}$, $G_{P} \triangleq g_{P} \tau$, and $G_{I} \triangleq g_{I} \tau^{2}$ fall within a restricted area, shown in Fig. 4. Since the system achieving the bandwidth max-min fairness was linear, the stability analysis could be carried out with the help of Nyquist stability criterion [21]. Secondly, with the help of the zero exclusion theorem [22], we have proven that the system with homogeneous delay $\bar{\tau}$ is asymptotically stable iff all systems with heterogeneous round-trip delays $\tau_{i} \leq \bar{\tau}$ are asymptotically stable. Moreover, the same stability is preserved even if the actual sum of flows' weights $w_{i}$ is smaller than the estimate, i.e., $\left|Q_{w}\right|<\left|\hat{Q}_{w}\right|$. $\left(w_{i},\left|Q_{w}\right|\right.$, and $\left|\hat{Q}_{w}\right|$ are defined in [19]. In our system, $w_{i}=1 \forall i \in Q$.) To summarize, this result implies the closed-loop system is asymptotically stable for $\mathcal{U}^{-1}(u)=h u$ and $h \in(0,1]$, that corresponds to letting $\left|\hat{Q}_{w}\right|=\left|Q_{w}\right| / h$ in [19].

Hence, we can see that $G(s) /(1+h G(s))$ is asymptotically stable for any gain sets $\left(G_{D}, G_{P}, G_{I}\right)$ falling within a restricted area shown in Fig. 4 , and $h \in(0,1]$. We are now ready to state the main result of this paper.

Theorem 2 (Homogeneous-Delay Case): The closed-loop system described by (1), (SA), and (LA1) [or (LA2)] with the homogeneous-delay assumption $\tau_{i}^{f}=\tau^{f}, \tau_{i}^{b}=\tau^{b} \forall i \in Q$, and $\tau^{f}+\tau^{b}=\tau$ is asymptotically stable for arbitrary utility functions with $0<k \leq d U_{i} / d a<\infty \forall a \in[0, \infty)$ and $\forall i \in Q$ if a gain set $\left(G_{D}, G_{P}, G_{I}\right)$ falls within a restricted area shown in Fig. 4, and there exist a finite number $\eta$ and a finite number $\theta \geq 0$ such that the open-loop transfer function $G(j \omega)$ satisfies the following equation for arbitrarily small $h>0$ :

$$
\operatorname{Re}\left[\left(1+\frac{j \omega \eta}{1+\theta \omega^{2}}\right) \frac{G(j \omega)}{1+h G(j \omega)}\right]+k>0 \quad \forall \omega \geq 0 .
$$

Remark 1: One should note that this requirement is not stringent, because the maximum slopes of the utility functions are not restricted, except for the condition that they should not be infinite. In other words, this restriction means that a user's satisfaction should increase with minimum slope of $k$ for the stability of the whole network. A user can sufficiently emphasize that his satisfaction increases significantly at a certain data rate with relatively high slope at that data rate; because what matters is not the absolute shape of one's utility function, but its relative shape compared with those of others.

Remark 2: If we multiply (8) by $\tilde{k} / k$, where $\tilde{k}$ is a positive number, it becomes

$$
\operatorname{Re}\left[\left(1+\frac{j \omega \eta}{1+\theta \omega^{2}}\right) \frac{\frac{\tilde{k}}{k} G(j \omega)}{1+\tilde{h} \frac{\tilde{k}}{k} G(j \omega)}\right]+\tilde{k}>0
$$

where $\tilde{h}=(k / \tilde{k}) h$. Then, we can observe from (9) that the modified open-loop transfer function $(\tilde{k} / k) G(j \omega)$ and the minimum slope $\tilde{k}$ satisfy Theorem 2 again. To restate this, if we use modified gain values $g_{P} \rightarrow(\tilde{k} / k) g_{P}, g_{I} \rightarrow(\tilde{k} / k) g_{I}$, and $g_{D} \rightarrow(\tilde{k} / k) g_{D}$, the minimum slope constraint becomes $d U_{i} / d a \geq \tilde{k}$. To sum up, if you want to replace the minimum slope $k$ with $\tilde{k}$, you can simply multiply stable gains by $\tilde{k} / k$.

The most effective aspect of this theorem is that utility functions have only the minimum slope requirement, and one user can use an arbitrarily shaped nonlinear utility function that may differ from the other users' utility functions. We strongly believe that our requirement is one of the least restrictive and most practical requirements in utility max-min network architecture.

We know from [19] that the closed-loop system is asymptotically stable when $U_{i}(a)=a$ for all $i \in N$. Thus we can infer that Theorem 2 is meaningful only when there exists $k \leq 1$ satisfying (8). Even though it is difficult to find a $k$ satisfying (8) for general cases, the inequality admits an intuitive graphical technique similar to the Nyquist stability criterion [21]. Let us define two functions $X$ and $Y$ as follows:

$$
\begin{aligned}
& X(\omega) \triangleq \operatorname{Re}\left[\frac{G(j \omega)}{1+h G(j \omega)}\right] \\
& Y(\omega) \triangleq \frac{\omega}{1+\theta \omega^{2}} \operatorname{Im}\left[\frac{G(j \omega)}{1+h G(j \omega)}\right] .
\end{aligned}
$$

Then (8) is equivalent to the following condition:

$$
\begin{gathered}
X(\omega)-\eta Y(\omega)+k>0 \quad \forall \omega \geq 0 \\
\Longleftrightarrow\left(\begin{array}{ll}
1 & -\eta
\end{array}\right)\left(\begin{array}{c}
X(\omega) \\
Y(\omega)
\end{array}\right)>-k \quad \forall \omega \geq 0 .
\end{gathered}
$$



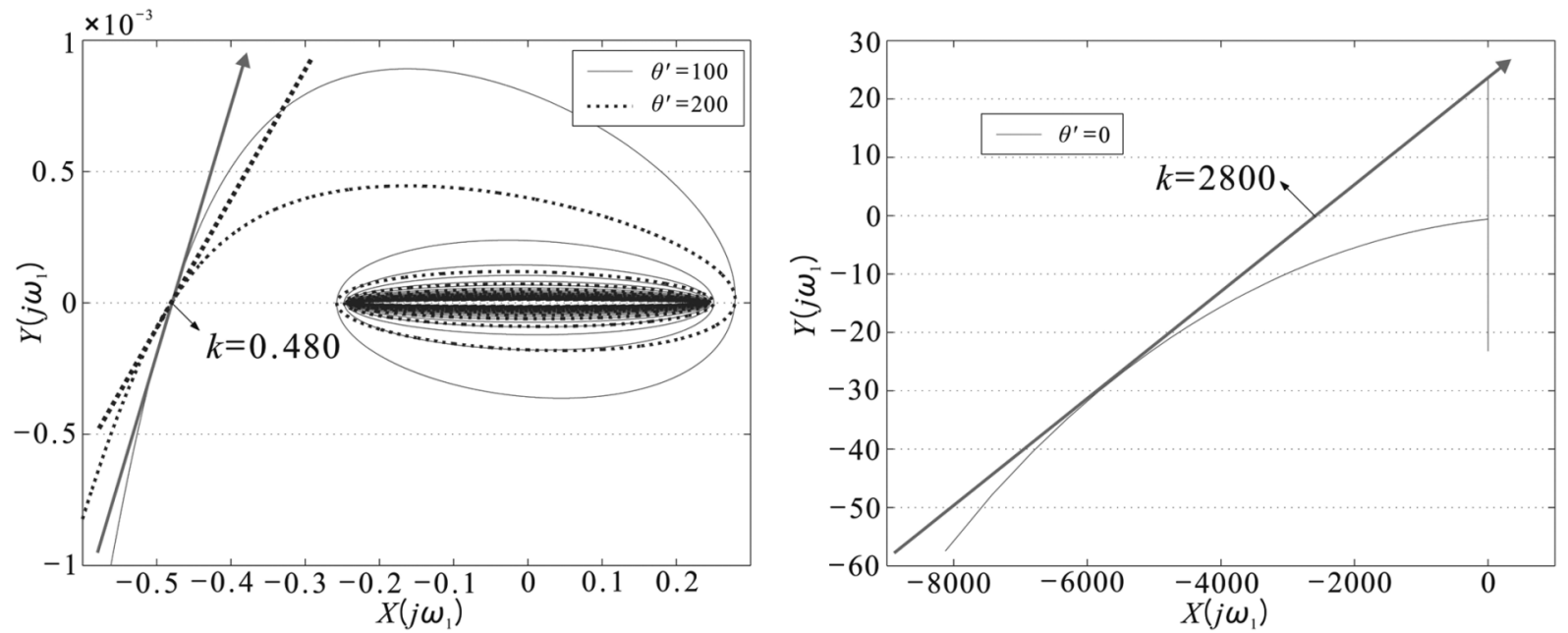

Fig. 5. Samples of $\left(X\left(\omega_{1}\right), Y\left(\omega_{1}\right)\right)$ plot for $G_{\text {PID }}^{3}(\rho=1)$ with $h=0.001$ and $\theta^{\prime}=0,100,200$.

In 2-D Euclidean space, this inequality entails that the closedloop system is asymptotically stable if there is a line with slope $1 / \eta$ and an intercept on the $X$ axis $-k$ such that $(X(\omega), Y(\omega))$ trajectory is completely contained in the open right half-space of the line. Because we have found that it is very difficult to find an equivalent and explicit symbolic expression of (8) independent of $\omega$, we provide minimum values of $k$ when two optimal gain sets found in [19] are used. The gain sets are optimal in the sense that they maximize the asymptotic decay rates of the closed-loop systems employing the identity utilities $U_{i}(a)=a$ $\forall i \in N$, whereas it is impossible even to define the asymptotic decay rates of nonlinear delayed systems. To find a $k$ value independent of $\tau$, we rewrite $G(j \omega), X(j \omega)$, and $Y(j \omega)$ into the following equations:

$$
\begin{aligned}
& G\left(j \omega_{1}\right)=-\frac{j \omega_{1} G_{P}+G_{I}-\omega_{1}^{2} G_{D}}{\omega_{1}^{2}} \\
& X\left(\omega_{1}\right) \triangleq \operatorname{Re}\left[\frac{G\left(j \omega_{1}\right)}{1+h G\left(j \omega_{1}\right)}\right] \\
& Y\left(\omega_{1}\right) \triangleq \frac{\omega_{1}}{1+\theta^{\prime} \omega_{1}^{2}} \operatorname{Im}\left[\frac{G\left(j \omega_{1}\right)}{1+h G\left(j \omega_{1}\right)}\right]
\end{aligned}
$$

where $\omega_{1} \triangleq \tau \omega$ and $\theta^{\prime} \triangleq \theta / \tau^{2}$.

Corollary 1 (Explicit Range of $k$ ): Let $G_{\mathrm{PID}}^{3} \triangleq$ $\left(G_{D}, G_{P}, G_{I}\right)=(0.242,0.868,0.261)$ and $G_{\mathrm{PID}}^{2} \triangleq$ $\left(G_{D}, G_{P}, G_{I}\right)=(0,0.482,0.091)$ that correspond to the PID and proportional-integral (PI) optimal gain sets, respectively. If $G_{\text {PID }}^{3} \times \rho$ and $G_{\text {PID }}^{2} \times \rho$ are used where $\rho>0$, the minimum values of $k$ are $0.480 \times \rho$ and $0.338 \times \rho$, respectively.

Remark 3: Note that for $\theta=0$ (or $\theta^{\prime}=0$ in Fig. 5), the inequality (8) reduces to the well-known Popov criterion where the minimum slope constraint is dropped. The minimum slope constraint in our theorem is essential for getting a smaller $k$, because the Popov criterion requires $k=2800$ for $G_{\mathrm{PID}}^{3}(\rho=$ $1)$ as shown in Fig. 5. Since $G_{\text {PID }}^{3}$ is optimal only for identity utilities $U_{i}(a)=a \forall i \in N$, a closed-loop system satisfying the sector condition, ${ }^{1}$ i.e., $U_{i}(a) / a \geq 2800 \forall i \in N$, converges at an extremely low speed. Thus, instead of the Popov criterion, which has been regarded as one of the least conservative criteria when the nonlinear feedback $\phi(\cdot)$ is time-invariant, we must use Dewey and Jury's criterion [20], which allows much smaller values of $k$ thanks to the minimum slope constraint.

This corollary provides minimum values of $k$ for two optimal gain sets. For the PID and PI controller models, respectively, with the gain set $G_{\mathrm{PID}}^{3} \times \rho$ and $G_{\mathrm{PID}}^{2} \times \rho$, we can use any kinds of utility function that satisfy, respectively, $0.480 \times \rho \leq d U_{i} / d a<$ $\infty$ and $0.338 \times \rho \leq d U_{i} / d a<\infty$. The astute reader might already have observed from Remark 1 that the linear relationship between the gain set and the minimum slope in Corollary 1 are much more important than the minimum slope itself. The point is how small the minimum slope can be for a given gain set. Thus, for brevity, we assume $\rho=1$ from now on. To introduce stability margins to the closed-loop system, it is recommended that the minimum slopes of utility functions be bounded by 1 .

Last, we would like to comment on the inevitability of the minimum-slope constraint in our distributed utility max-min architecture, represented by (1), (SA), and (LA1). In a single-link case, (SA) reduces to $a_{i}(t)=U_{i}^{-1}\left(u\left(t-\tau_{i}^{b}\right)\right)$. If the slope $d U_{i} / d u$ is sufficiently small, a small change in $u(t)$ can induce a large fluctuation in $a_{i}(t)$. Since feedback delays between $a_{i}(t)$ and $u(t)$ are nonzero, the fluctuation of $a_{i}(t)$ is not alleviated immediately and causes the instability of the queue length through (1). Thus, we can see that the minimum-slope constraint of utility functions is inevitable in a distributed utility max-min architecture with nonzero feedback delays.

\section{MiscellaneOUS TOPICS}

\section{A. Estimation of $\left|Q^{l}\right|$}

The usage of $\left|Q^{l}\right|$, the number of flows bottlenecked at link $l$ in (LA1) and (LA2), is not a specific requirement of our architecture but an inevitable requirement of most approaches.

${ }^{1}$ Popov criterion requires the sector condition, i.e., $U_{i}(a) / a \geq k$, while Dewey and Jury's criterion [20] requires the incremental sector condition, i.e., $d U_{i}(a) / d a \geq k$. For a more detailed explanation, see [23]. 


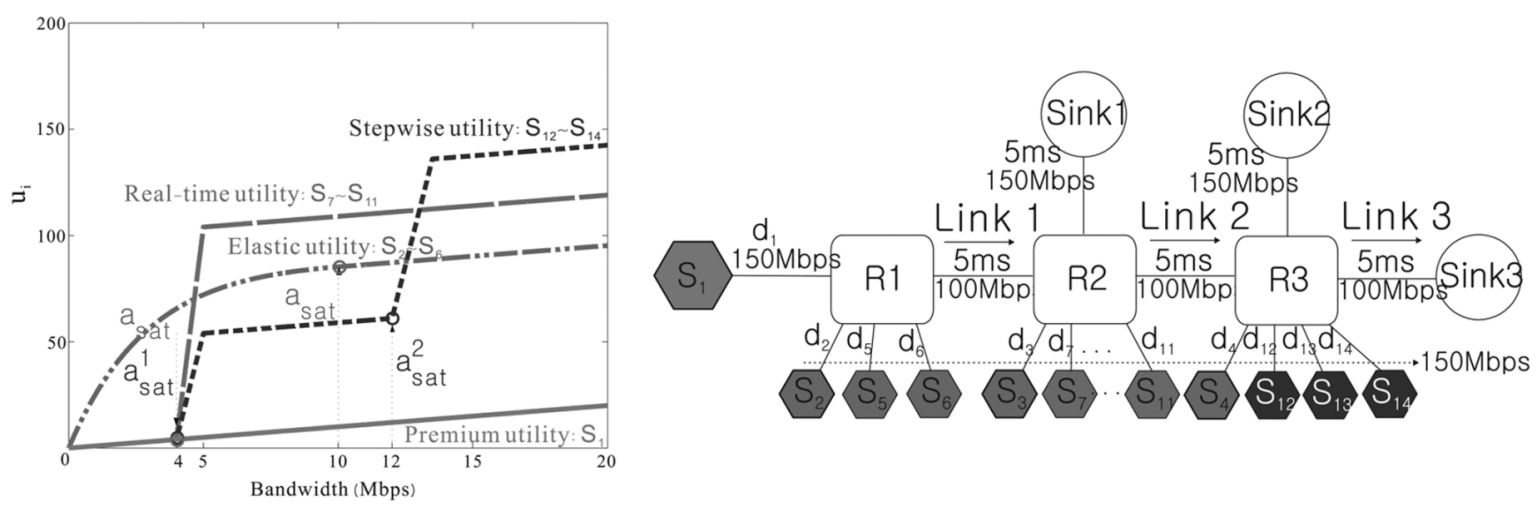

Fig. 6. Utility functions for different application classes and multiple-bottleneck network used for simulation.

In most approaches, the gain value used for updating source rates or link prices is normalized by analogous terms. However, routers should store per-flow information regarding flows' activeness to know the exact value of $\left|Q^{l}\right|$. Thus, to eliminate this overhead, $\left|Q^{l}\right|$ must be estimated properly. The condition of (12) is still satisfied when $|Q|$ in (7) is replaced by $|\hat{Q}| \geq|Q|$. Thus, we can see that the overestimation $\left|Q^{l}\right|$ is allowed, while severe overestimation slows down the convergence speed of the closed-loop system.

We largely follow the method described in [5] and modify the method for utility max-min fairness. When the $j$ th data packet arrives at link $l$ at time $t^{j}$, it contains two fields, $a_{i}\left(t^{j}-\tau_{i}^{l, f}\right)$ and $u^{l^{\prime}}\left(t^{j}-\tau_{i}^{l^{\prime}, f}\right)$ where $l^{\prime}$ is the current bottleneck link for flow $i$. Using these two values, for the $k$ th interval, the number of flows bottlenecked at link $l$ can be approximated by

$$
\begin{aligned}
&\left|\hat{Q}^{l}\right|^{k}=\sum_{t^{j} \in((k-1) W, k W]} \frac{\operatorname{DPS}^{j}}{W \cdot a_{i}\left(t^{j}-\tau_{i}^{l, f}\right)} \\
& \cdot 1\left\{u^{l^{\prime}}\left(t^{j}-\tau_{i}^{l^{\prime}, f}\right) \geq \delta \cdot u^{l}\left(t^{j}\right)\right\}
\end{aligned}
$$

where $1\{\cdot\}$ is the indicator function, $W$ is the time interval used for averaging, and $\mathrm{DPS}^{j}$ is the size of the $j$ th data packet. When $u^{l^{\prime}}\left(t^{j}-\tau_{i}^{l^{\prime}, f}\right) \geq \delta \cdot u^{l}\left(t^{j}\right)$, flow $i$ is regarded a flow bottlenecked at link $l$ and the indicator function returns 1 , otherwise 0 . The value $\delta=0.9$ is used to introduce a margin for estimation. Note that (11) provides an efficient method for estimating $\left|Q^{l}\right|$ in addition to preventing underestimation of $\left|Q^{l}\right|$. For suppression of the fluctuation in estimation, the value $\left|\hat{Q}^{l}\right|$ is computed as follows:

$$
\left|\hat{Q}^{l}\right| \leftarrow \max \left[1, \lambda\left|\hat{Q}^{l}\right|+(1-\lambda)\left|\hat{Q}^{l}\right|^{k}\right]
$$

where $\lambda$ is an averaging factor, and it is found that $\lambda$ yields the stable and effective estimation of $\left|\hat{Q}^{l}\right|$ when it is set to $\lambda=0.98$.

\section{B. Applicability of Our Work}

Recently, Tang et al. [24] showed that max-min fairness achieves higher aggregate throughput than proportional fairness [7]-[9] in some cases. Thus, as of now, no one can say that one is better than the other. Sarkar and Tassiulas [12] also argued that max-min fairness might be a fairer choice than proportional fairness from the user's viewpoint, because max-min fairness treats flows equally irrespective of their path length, whereas proportional fairness penalizes flows traversing longer paths. Since the same argument applies to utility-based fairness concepts, it can be asserted that the stability analysis of distributed utility max-min flow control is also important.

To support various multimedia applications in multirate multicast networks, Rubenstein et al. [15] employed utility max-min fairness. They noted that different network applications can have differing bandwidth requirements to support a given level of quality for an application. They showed that if multicast sessions are multirate, the utility max-min fair allocation satisfies desirable fairness properties that do not hold in a single-rate utility max-min fair allocation. Bianchi et al. [10] emphasized that the scalable properties of multimedia content should be exploited when the desired resources are only partially available, and motivated the need of adaptive QoS techniques in wireless networks. As an adaptation technique, they adopted equal utility allocation. After due consideration of these works, we believe that our distributed link algorithms, which achieve the utility max-min fairness with guaranteed stability, will facilitate the application of utility-based fairness concepts.

\section{Simulation Results}

We provide several simulation results using the ns- 2 simulator [25] to demonstrate the merits of utility max-min flow control and the performance of our algorithms. The largest round-trip propagation delays are set to $100 \mathrm{~ms}$. To avoid messy figures, we simulated our architecture with only two kinds of three-term link controllers, i.e., PID and $\mathrm{PII}^{2}$ controllers. Simulation results for the PID and $\mathrm{PII}^{2}$ link controller models are, respectively, denoted by $G_{\mathrm{PID}}^{3}$ and $G_{\mathrm{PII}^{2}}^{3}$. For two-term link controllers, i.e., $\mathrm{PI}$ and $\mathrm{PII}^{2}$ controllers, we can obtain simulation results similar to those given in [19].

Four utilities, i.e., premium, elastic, real-time, and stepwise utilities, are approximated by slope-restricted utility functions in Fig. 6. To satisfy the minimum slope constraint and to introduce stability margins, all utilities in Fig. 6 are designed to satisfy $d U_{i} / d u(a) \geq 1$. It should be remarked that the value of $U_{i}(a)$ has no specific meaning, and its unit can be arbitrarily chosen by introducing some scaling factor in (LA1) and (LA2).

To show that the proposed models work well in multiple-bottleneck networks, we consider a network configuration in which 
TABLE I

Flow MODELS USED FOR SIMULATION

\begin{tabular}{l||crrrr}
\hline source & utility & $\mathrm{d}_{\mathrm{i}}$ & begin(s) at & end(s) at & sink \\
\hline $\mathrm{S}_{1}$ & premium & $35 \mathrm{~ms}$ & $-\infty$ & $\infty$ & Sink3 \\
$\mathrm{S}_{2}$ & elastic & $15 \mathrm{~ms}$ & $-\infty$ & $\infty$ & Sink1 \\
$\mathrm{S}_{3}$ & elastic & $20 \mathrm{~ms}$ & $-\infty$ & $\infty$ & Sink2 \\
$\mathrm{S}_{4}$ & elastic & $5 \mathrm{~ms}$ & $-\infty$ & $\infty$ & Sink3 \\
$\mathrm{S}_{5}, \mathrm{~S}_{6}$ & elastic & $25,30 \mathrm{~ms}$ & $10,10.1 \mathrm{~s}$ & $\infty$ & Sink1 \\
$\mathrm{S}_{7}, \mathrm{~S}_{8}, \mathrm{~S}_{9}, \mathrm{~S}_{10}, \mathrm{~S}_{11}$ & real-time & $20,40,15,40,25 \mathrm{~ms}$ & $20,20.1,20.2,20.3,20.4 \mathrm{~s}$ & $\infty$ & Sink2 \\
$\mathrm{S}_{12}, \mathrm{~S}_{13}, \mathrm{~S}_{14}$ & stepwise & $30,40,10 \mathrm{~ms}$ & $40,40.1 \mathrm{~s}$ & $\infty$ & Sink3 \\
\hline
\end{tabular}
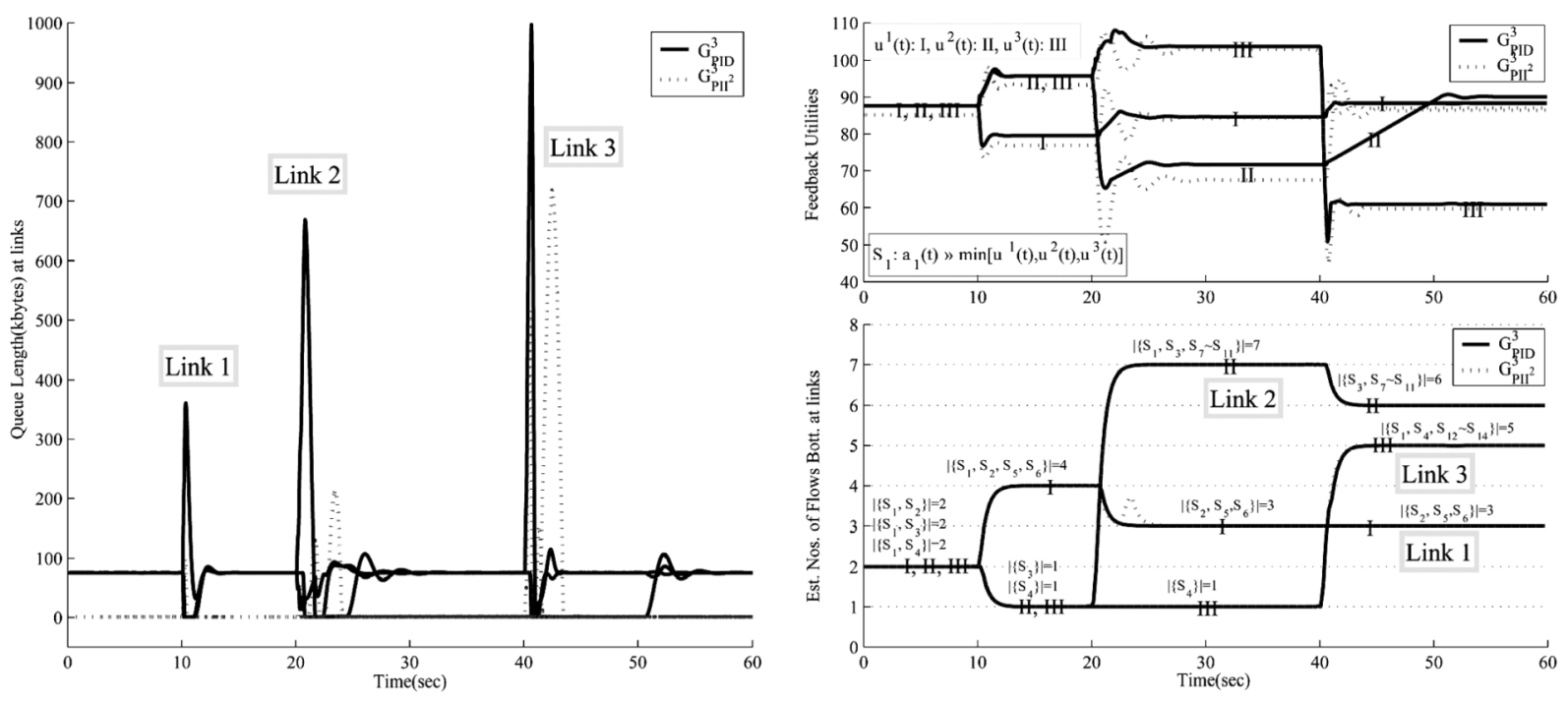

(a) Queue length at links $\left(q^{l}(t)\right)$, Feedback utilities at links $\left(u^{l}(t)\right.$ ), Source sending rate of $\mathrm{S}_{1}\left(a_{1}(t)\right)$ and Estimated numbers of flows bottlenecked at links $\left(\left|\hat{Q}^{l}\right|\right)$.
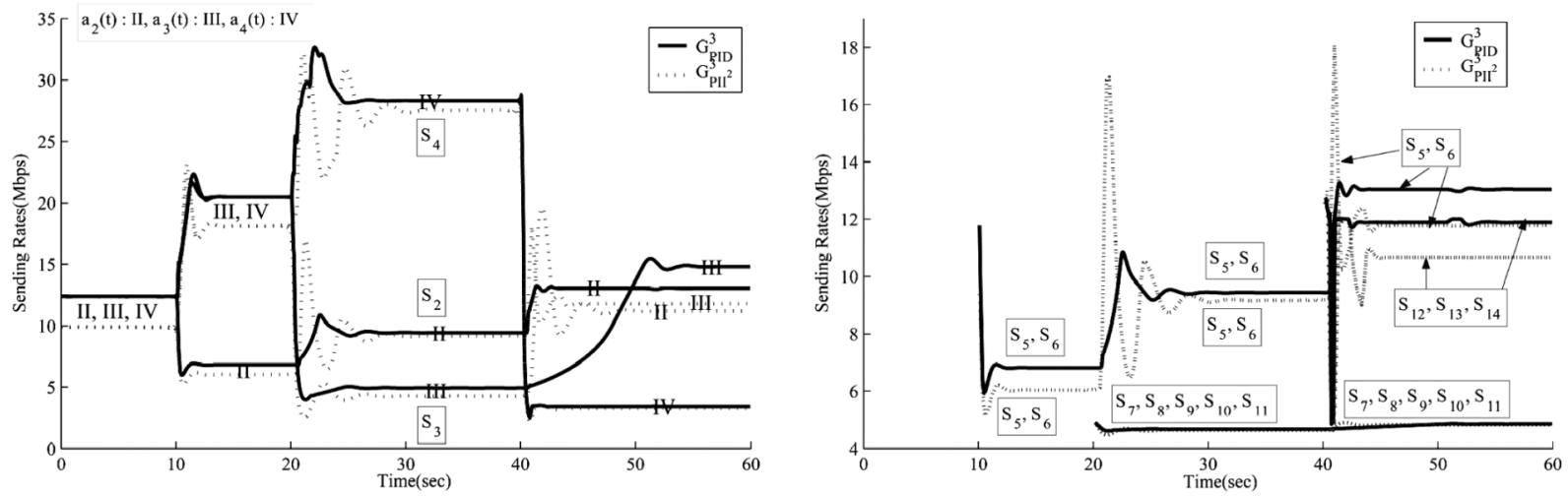

(b) Source sending rates of $\mathrm{S}_{2} \sim \mathrm{S}_{14}\left(a_{2}(t) \sim a_{14}(t)\right)$.

Fig. 7. Simulation results.

there are three bottleneck links; see Fig. 6, where $\bar{\tau}=120 \mathrm{~ms}$ is used. The flow models are summarized in Table I. In Fig. 7(a), although there are queue overshoots at $t=10,20$, and $40 \mathrm{~s}$ because several flows begin transmission simultaneously, such dramatic events (e.g., $S_{7} \sim S_{11}$ begin transmission simultaneously) do not occur frequently in real networks. In steady states, the sending rates of flows satisfy the feasibility condition in Definition 1 and the utility max-min property in Definition 2, as shown in Fig. 7(b). For the PID model, the link capacity is fully used, while the target queue length $\left(q_{T}^{l}=75 \mathrm{kB}\right)$ is obtained. For the $\mathrm{PII}^{2}$ model, the target use $\left(\alpha_{T}^{l}=0.95\right)$ is achieved, while the queue length at steady state becomes zero. Because $S_{1}$ traverses links 1,2 , and $3, a_{1}(t)$ becomes nearly identical to the minimum of the feedback utilities at the three links, $\min \left[u^{1}(t), u^{2}(t), u^{3}(t)\right]$.

Four intervals are readily distinguishable; $[-\infty, 10 \mathrm{~s}],[10 \mathrm{~s}$, $20 \mathrm{~s}],[20 \mathrm{~s}, 40 \mathrm{~s}]$, and $[40 \mathrm{~s}, \infty]$. From $-\infty$ to $t=10 \mathrm{~s}, S_{1}$ is bottlenecked at all three links. As new elastic flows, $S_{5}$ and $S_{6}$ destined for Sink1 begin transmission at $t=10 \mathrm{~s}, S_{1}$ becomes bottlenecked only at link 1 . Thus from $t=10 \mathrm{~s}$ to $t=20 \mathrm{~s}$, 
flows $S_{3}$ and $S_{4}$ can send data at higher rates, and $S_{2}$ can send data at a lower rate compared with the previous time interval, as shown in Fig. 7(b). As five real-time flows, $S_{7} \sim S_{11}$ destined for Sink2 begin transmission at $t=20 \mathrm{~s}, S_{1}$ is now bottlenecked at link 2. From $t=20 \mathrm{~s}$ to $t=40 \mathrm{~s}, S_{2}, S_{5}$, and $S_{6}$ can send data at higher rates, and $S_{3}$ can send data at a lower rate compared with the previous time interval. Similarly, when three stepwise flows $\left(S_{12} \sim S_{14}\right)$ destined for Sink3 begin transmission at $t=$ $40 \mathrm{~s}, S_{1}$ becomes bottlenecked at link 3 and flows are allocated bandwidth according to utility max-min fairness. From $t=40 \mathrm{~s}$ to $t=60 \mathrm{~s}$, link 1 becomes lightly congested compared with link 3. Thus two elastic flows traversing link $1, S_{5} \sim S_{6}$, can send data at higher rates than three stepwise flows traversing link 3, $S_{12} \sim S_{14}$ can. We can verify that our proposed algorithms work well in multiple-bottleneck networks, where the bottleneck link of a flow can change dynamically as the network situation changes.

The estimated numbers of flows bottlenecked at links, $\left|\hat{Q}^{l}\right|$, nicely trace the exact numbers of flows bottlenecked at links, $\left|Q^{l}\right|$, in steady states. For example, the flows bottlenecked at link 3 are $\left\{S_{1}, S_{4}\right\},\left\{S_{4}\right\}$, and $\left\{S_{1}, S_{4}, S_{12}, S_{13}, S_{14}\right\}$, respectively, in the intervals $[-\infty, 10 \mathrm{~s}],[10 \mathrm{~s}, 40 \mathrm{~s}]$, and [40 s, $\infty]$. Thus, $\left|\hat{Q}^{3}\right|$ becomes 2, 1, and 5, respectively, as shown in Fig. 7(a). If $\left|\hat{Q}^{l}\right|$ underestimates $\left|Q^{l}\right|$ for a short time of transient periods as shown in Fig. 7(a), the effective gains become $\left|Q^{l}\right| /\left|\hat{Q}^{l}\right|$ times the original values and become larger than the original gains. These enlarged gains can cause an adverse effect to the stability of the network when the number of flows in the network is relatively small, whereas reduced gains only slow down the convergence of the network. Thus, one can intentionally lower-bound $\left|\hat{Q}^{l}\right|$ by a positive integer value to cope with this situation.

\section{CONCLUDING REMARKS}

We have proposed a control-theoretic framework for application performance-oriented flow control. Our contribution is three-fold. First, we have found a distributed-link algorithm that attains utility max-min bandwidth sharing while controlling link buffer occupancy to either zero or a target value. Moreover, the link algorithm does not require any per-flow information and processing, so it is scalable. Second, our algorithm is shown to be asymptotically stable in the presence of round-trip delays for arbitrary forms of utility function, as long as they are continuous and their slopes are larger than a certain positive constant. Third, our framework lends itself to a single unified flow-control scheme that can simultaneously serve not only elastic flows, but also nonelastic flows such as voice, video, and layered video.

\section{APPENDIX I \\ PROOF OF THEOREM 1}

If all links in the network perform the same operation as that described in Section II, each flow has its own bottleneck link (which can be more than one), and the utility vector at steady state is feasible in the sense that it satisfies (5) for all bottleneck links $l \in L$. If we increase the utility value of flow $i$ which is bottlenecked at some link $l$ while maintaining feasibility, we should reduce the data rate of flow $i^{\prime}(\neq i)$ that traverses link $l$, i.e., $i^{\prime} \in N(l)$. Since $U_{i^{\prime}}(\cdot)$ is an increasing function by the assumption A.1, the utility value of $i^{\prime}$ is also reduced. Since
$U_{i^{\prime}}\left(a_{i^{\prime}}^{*}\right) \operatorname{leq} U_{i}\left(a_{i}^{*}\right)$ for all $i^{\prime} \in N(l)$ by the definition of bottleneck link (see, e.g., [1]), we are reducing the utility value of flow $i^{\prime}$, which is already less than or equal to the utility value of flow $i$. This complete the proof. To the PII ${ }^{2}$ model, a similar proof is applicable.

\section{APPENDIX II \\ PROOF OF THEOREM 2}

For notational simplicity, we define two functions shown in Fig. 3(b) as follows:

$$
\left.\bar{G}(s) \triangleq \frac{G(s)}{(1+h G(s)}\right), \quad \overline{\mathcal{U}}^{-1}(u) \triangleq \mathcal{U}^{-1}(u)-h u .
$$

By the assumption that $\left(G_{D}, G_{P}, G_{I}\right)$ is contained in Fig. 4, we can see that $\bar{G}(s)$ is asymptotically stable for any $h \in(0,1]$ from the arguments of $[19$, Sec. 3.3]. Then we can apply Dewey and Jury's criterion ([20, Corollary 5]) to our nonlinear monotone feedback system, because $\bar{G}(s)$ is asymptotically stable so that $g(t)$ and $\dot{g}(t)$ become elements of $L_{1}(0, \infty)$, i.e., the set of absolutely integrable functions and $\overline{\mathcal{U}}^{-1}(0)=0$ by the assumption A.2. Although the differentiability of feedback nonlinearities was also assumed, this assumption is used only for the simplicity of their proof. If the feedback nonlinearities have left-hand and right-hand derivatives at all points, Dewey and Jury's criterion still holds.

If there exist a finite number $\eta$ and a finite number $\theta \geq 0$ such that the inequality (8) is satisfied for some small $h>0$, then the closed-loop system is asymptotically stable with $\mathcal{U}^{-1}(u)$ satisfying the following equation by Dewey and Jury's criterion:

$$
0 \leq \frac{d}{d u}\left(\mathcal{U}^{-1}(u)-h u\right) \leq \frac{1}{k}
$$

If this is satisfied for arbitrarily small $h>0$, we have the following condition for $\mathcal{U}^{-1}(u)$ :

$$
0<\frac{d \mathcal{U}^{-1}(u)}{d u} \leq \frac{1}{k}
$$

When each of the utility functions $U_{i}(a)$ satisfies $k \leq$ $d U_{i} / d a<\infty$, then it also satisfies $0<d U_{i}^{-1} / d u \leq 1 / k$ and their sum becomes as follows, due to the finitude of $|Q|$ :

$$
0<\frac{1}{|Q|} \sum_{i \in Q} \frac{d U_{i}^{-1}(u)}{d u} \leq \frac{1}{k} \Longleftrightarrow 0<\frac{d \mathcal{U}^{-1}(u)}{d u} \leq \frac{1}{k} .
$$

Therefore, we can conclude that the closed-loop system is asymptotically stable if the minimum slope of the utility functions is restricted by $k$, i.e., $k \leq d U_{i} / d a<\infty$ for all $a \in[0, \infty)$. For the $\mathrm{PII}^{2}$ model, we can apply the same procedure because $G(s)$ of the $\mathrm{PII}^{2}$ model is identical to that of the PID model.

\section{APPENDIX III \\ PROOF OF COROLLARY 1}

Let us assume $\rho=1$. To apply Theorem 2, the condition of (10) should be satisfied for $h$ arbitrarily close to 0 . When we use a graphical technique with small $h, \theta^{\prime}$ has an essential role in achieving a smaller $k$. The inequality (10) implies that $\theta^{\prime}$ has no significant effect when $\omega_{1}$ is small, and in that 
case, the $\left(X\left(\omega_{1}\right), Y\left(\omega_{1}\right)\right)$ trajectories are nearly identical, independent of $\theta^{\prime}$. However, when $\omega_{1}$ is sufficiently large and $\theta^{\prime}$ is very small, $Y\left(\omega_{1}\right)$ becomes very large, and the trajectories of $\left(X\left(\omega_{1}\right), Y\left(\omega_{1}\right)\right)$ prevents us from obtaining a smaller $k$ value. Using the graphical technique introduced in Section III, we can show that the following $k$ values satisfy $X\left(\omega_{1}\right)-\eta^{\prime} Y\left(\omega_{1}\right)+k>$ $0 \forall \omega_{1} \geq 0$ for some $\eta^{\prime} \triangleq \eta / \tau$ and $\theta^{\prime} \geq 0$ :

$$
k=\frac{G_{P}}{\omega_{1}^{*} \sin \omega_{1}^{*}}+\epsilon
$$

where $\omega_{1}^{*}$ is the smallest $\omega_{1}>0$ satisfying $\left(G_{I}-\right.$ $\left.\omega_{1}^{2} G_{D} / \omega_{1}^{2}\right) S \in\left(\omega_{1}\right)-\left(G_{P} / \omega_{1}\right) \cos \left(\omega_{1}\right)=0$ and $\epsilon>0$ is any finite number. For $G_{\mathrm{PID}}^{3}$ and $G_{\mathrm{PID}}^{2}$, values of $\left(G_{P} / \omega_{1}^{*}\right) \sin \omega_{1}^{*}$ are approximately 0.4798 and 0.3375 , respectively. For the proof of the case $\rho \neq 1$, refer to Remark 2. This completes the proof.

\section{ACKNOWLEDGMENT}

The authors are grateful to the anonymous reviewers whose insightful and constructive comments significantly improved the quality of this work.

\section{REFERENCES}

[1] D. Bertsekas and R. Gallager, Data Networks. Englewood Cliffs, NJ: Prentice-Hall, 1992.

[2] B. Radunović and J.-L. LeBoudec, "A unified framework for max-min and min-max fairness with applications," in Proc. Annu. Allerton Conf., 2002, CD-ROM.

[3] L. Benmohamed and S. Meerkov, "Feedback control of congestion in packet switching networks: The case of single congested node," IEEE/ACM Trans. Netw., vol. 1, no. 6, pp. 693-708, Dec. 1993.

[4] L. Benmohamed and S. Meerkov, "Feedback control of congestion in packet switching networks: The case of multiple congested node," Int. J. Commun. Syst., vol. 10, no. 5, pp. 227-246, 1997.

[5] S. Chong, S. H. Lee, and S. H. Kang, "A simple, scalable, and stable explicit rate allocation algorithm for max-min flow control with minimum rate guarantee," IEEE/ACM Trans. Netw., vol. 9, no. 3, pp. 322-335, Jun. 2001.

[6] F. Blanchini, R. L. Cigno, and R. Tempo, "Robust rate control for integrated services packet networks," IEEE/ACM Trans. Netw., vol. 10, no. 5 , pp. 644-652, Oct. 2002.

[7] F. Kelly, "Charging and rate control for elastic traffic," Eur. Trans. Telecommun., vol. 8, pp. 33-37, 1997.

[8] F. Kelly, A. Maulloo, and D. Tan, "Rate control for communication networks: Shadow prices, proportional fairness and stability," J. Oper. Res. Soc., vol. 49, no. 3, pp. 237-252, Mar. 1998.

[9] S. H. Low and D. E. Lapsley, "Optimization flow control—Part I: Basic algorithms and convergence," IEEE/ACM Trans. Netw., vol. 7, no. 4, pp. 861-875, Aug. 1999.

[10] G. Bianchi, A. T. Campbell, and R. R.-F. Liao, "On utility-fair adaptive services in wireless networks," Proc. IEEE/IFIP IWQoS, pp. 256-267, 1998.

[11] Z. Cao and E. W. Zegura, "Utility max-min: An application-oriented bandwidth allocation scheme," Proc. IEEE INFOCOM, pp. 793-801, 1999.

[12] S. Sarkar and L. Tassiulas, "Fair allocation of utilities in multirate multicast networks: A framework for unifying diverse fairness objectives," IEEE Trans. Autom. Control, vol. 47, no. 6, pp. 931-944, Jun. 2002.
[13] T. Harks, "Utility proportional fair bandwidth allocation: An optimization oriented approach," in Proc. QoS Multiservice IP Netw., Springer LNCS 3375, 2005, pp. 61-74.

[14] T. Harks and T. Poschwatta, "Utility fair congestion control for realtime traffic," in Proc. IEEE Global Internet Symp./IEEE INFOCOM, 2005, pp. 2786-2791.

[15] D. Rubenstein, J. Kurose, and D. Towsley, "The impact of multicast layering on network fairness," IEEE/ACM Trans. Netw., vol. 10, no. 2, pp. 169-182, Apr. 2002.

[16] B. Wydrowski, L. L. H. Andrew, and M. Zukerman, "Maxnet: A congestion control architecture for scalable networks," IEEE Commun. Lett., vol. 7, no. 10, pp. 511-513, Oct. 2003.

[17] H. K. Khalil, Nonlinear Systems. Upper Saddle River, NJ: PrenticeHall, 2002.

[18] J. K. Hale and S. M. V. Lunel, Introduction to Functional Differential Equations. London, U.K.: Springer-Verlag, 1993.

[19] J. Cho and S. Chong, "Stabilized max-min flow control using PID and $\mathrm{PII}^{2}$ controllers," IEICE Trans. Commun., vol. E88-B, no. 8, pp. 3353-3364, Aug. 2005.

[20] A. G. Dewey and E. I. Jury, "A stability inequality for a class of nonlinear feedback systems," IEEE Trans. Autom. Control, vol. AC-11, no. 1, pp. 54-62, Jan. 1966.

[21] R. C. Dorf and R. H. Bishop, Modern Control Systems. Reading, MA: Addison-Wesley, 1995.

[22] J. Ackermann, Robust Control: Systems With Uncertain Physical Parameters. New York: Springer-Verlag, 1993.

[23] M. Vidiyasagar, Nonlinear Systems Analysis. Englewood Cliffs, NJ: Prentice-Hall, 1993.

[24] A. Tang, J. Wang, and S. H. Low, "Is fair allocation always inefficient," in Proc. IEEE INFOCOM, 2004, pp. 35-45.

[25] UCB/LBNL/VINT Network Simulator-NS. ver. 2.1b9a.

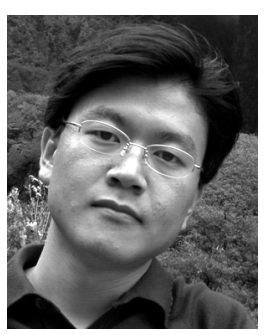

Jeong-woo Cho (S'01-M'06) received the B.S., M.S., and Ph.D. degrees in electrical engineering and computer science from the Korea Advanced Institute of Science and Technology (KAIST), Daejeon, Korea, in 2000, 2002, and 2005, respectively.

From September 2005, he has been with the Telecommunication R\&D Center, Samsung Electronics, Suwon, Korea, as a Senior Engineer. His research interests are focused on the area of flow control, congestion control, scheduling, and power control in wired/wireless networks.

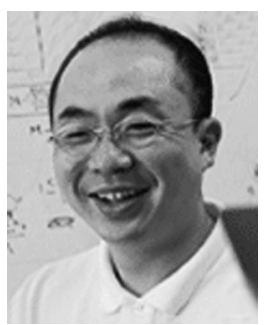

Song Chong (S'93-M'95) received the B.S. and M.S. degrees in control and instrumentation engineering from Seoul National University, Seoul, Korea, in 1988 and 1990, respectively, and the Ph.D. degree in electrical and computer engineering from the University of Texas at Austin in 1995.

Since March 2000, he has been with the School of Electrical Engineering and Computer Science, Korea Advanced Institute of Science and Technology (KAIST), Daejeon, Korea, where he is an Associate Professor, leading the Network Systems Laboratory. Prior to joining KAIST, he was with the Performance Analysis Department, AT\&T Bell Laboratories, Holmdel, NJ, as a Member of Technical Staff. His research interests include high-speed networks, wireless networks and performance evaluation. He has published more than 60 papers in international journals and conferences and holds three U.S. patents in these areas.

Dr. Chong is an Editor of the Journal of Communications and Networks, and has served as a Technical Program Committee member of numerous international conferences. 